Mots. Les langages du politique

Trente ans d'étude des langages du politique

(1980-2010)

\title{
Développement et diversification d'une onomastique politique
}

\section{Paul Bacot}

\section{OpenEdition}

\section{Journals}

Édition électronique

URL : https://journals.openedition.org/mots/19859

DOI : $10.4000 /$ mots. 19859

ISSN : 1960-6001

Éditeur

ENS Éditions

\section{Édition imprimée}

Date de publication : 30 novembre 2010

Pagination : 47-56

ISBN : 978-2-84788-235-3

ISSN : 0243-6450

\section{Référence électronique}

Paul Bacot, « Développement et diversification d'une onomastique politique », Mots. Les langages du politique [En ligne], 94 | 2010, mis en ligne le 06 novembre 2012, consulté le 28 avril 2022. URL : http:// journals.openedition.org/mots/19859; DOI : https://doi.org/10.4000/mots.19859 


\section{Mots \\ Les langages du politique}

№ 94 novembre 2010

\section{Trente ans d'étude des langages du politique (1980-2010)}

ouvrage coordonné par Paul BACOT, Marlène Coulomb-GulLY, Jean-Paul Honoré, Christian Le BART, Claire Oger, Christian Plantin

\section{SOMMAIRE}

Paul Bacot, Marlène Coulomb-Gully, Jean-Paul Honoré, Christian Le Bart, Claire Oger, Christian Plantin

Le discours politique n'est pas transparent. Permanence

et transformations d'un objet de recherche

OUTILS ET ENJEUX DU DISCOURS POLITIQUE

Ruth Amossy, Roselyne Koren

Argumentation et discours politique

Christian Plantin

Argumentation-rhétorique. Les eaux mêlées

Caroline Ollivier-Yaniv

Discours politiques, propagande, communication, manipulation 
Marc Bonhomme

La caricature politique

Paul Bacot

Développement et diversification d'une onomastique politique

Ruth Wodak

The Discursive Construction of History. Brief Considerations

Henri Boyer

Les politiques linguistiques

\section{LIEUX DE LA PRODUCTION DU DISCOURS POLITIQUE}

Christian Le Bart

Parler en politique

Dominique Maingueneau

Le discours politique et son «environnement»

Alice Krieg-Planque, Claire Oger

Discours institutionnels. Perspectives pour les sciences

de la communication

Sophie Béroud, Josette Lefèvre

Le corpus syndical. Une expérience au long cours

Corinne Gobin, Jean-Claude Deroubaix

L'analyse du discours des organisations internationales.

Un vaste champ encore peu exploré

François de la Bretèque

Le retour de la parole politique dans le cinéma français

Jean-Claude Soulages

Vie et mort du citoyen cathodique

Jacques Guilhaumou

Les discours de la Révolution française.

Aperçu d'ensemble d'un trajet de recherche (1980-2009) 


\section{DISCIPLINES ET CHAMPS DE RECHERCHE POUR L'ÉTUDE DES LANGAGES DU POLITIQUE}

Philippe Braud

L'apport de la science politique à l'étude des langages du politique

Claire Blandin

L'apport de l'histoire des médias à l'étude des langages du politique

Jean-François Tétu, Bernard Lamizet

Les SIC et les langages du politique

Sylvianne Rémi-Giraud

Sémantique lexicale et langages du politique.

Le paradoxe d'un mariage difficile?

Marlène Coulomb-Gully, Juliette Rennes

Genre, politique et analyse du discours.

Une tradition épistémologique française gender blind

Johannes Angermüller

Analyser le discours politique en Allemagne (1980-2010)

Érik Neveu

L'apport de Pierre Bourdieu à l'analyse du discours.

D'un cadre théorique à des recherches empiriques

Roselyne Ringoot

Questionner le discours avec Michel Foucault.

Actualisations théoriques et actualité éditoriale

\section{ENTRETIEN}

Maurice Tournier

Mots et politique, avant et autour de 1980

La revue Mots. Les langages du politique encourage l'usage des rectifications de l'orthographe proposées par le Conseil supérieur de la langue française et approuvées par l’Académie (Journal officiel, $n^{\circ}$ 100, 6 décembre 1990). 


\section{Développement et diversification d'une onomastique politique}

Si l'onomastique a toujours intéressé les linguistes, ceux-ci s'en sont longtemps tenus à l'étude des seuls noms de lieux (toponymes), de personnes (anthroponymes) et de peuples (ethnonymes). La science politique, quant à elle, a jusqu'à une date récente très largement délaissé ce champ de recherche. L'onomastique politique a pourtant toute sa place dans une politologie lexicale elle-même indissociable des autres approches de la sociologie du politique. Dans cette perspective, nombreux sont, en sciences du langage, les travaux indispensables à la compréhension du fait dénominatif en matière politique. On constate que les noms propres pris en exemple ou les problématiques qui leur sont appliquées relèvent souvent de l'analyse politique - surtout si l'on ne s'en tient pas à la politique entendue comme un ensemble particulier d'acteurs, d'institutions et de terrains ainsi étiquetés dans le discours courant, mais que l'on prend en considération le politique, entendu comme une mise en scène de la conflictualité sociale.

C'est dans cette optique qu'a été produite récemment une première recension, nécessairement non exhaustive, de la production francophone traitant d'onomastique dans une perspective susceptible d'intéresser directement ou indirectement les spécialistes de l'étude des langages du politique (Bacot, Douzou, Honoré, 2008).

\section{L'élargissement du champ d'investigation}

On aura une idée de la grande variété des noms propres en politique à travers la lecture d'une partie des notices contenues dans certains ouvrages de la collection «Les mots de... » ouverte en 2001 aux Presses du Mirail, comme notamment ceux sur la colonisation (Dulucq, Klein, Stora, 2008), la République (Agulhon, 2007) et Mai 1968 (Tournier, 2007), des Lieux de mémoire (Nora, 1984, 1986, 1992), ou des résumés des contributions au Séminaire d'onomastique politique initié à Lyon en $2008^{1}$.

1. Voir: [http://triangle.ens-Ish.fr/spip.php?rubrique283]. 
Traditionnellement, on considère que le nom propre (en général) permet de répondre aux questions qui? (anthroponymes) et où? (toponymes), auxquelles en toute logique il conviendrait d'ajouter quand? Dit autrement, le nom propre renvoie aux déictiques je, ici, maintenant (Van de Velde, 2000). La consultation de la collection de la Nouvelle revue d'onomastique confirmera le tropisme de cette discipline pour les deux premières catégories.

De fait, la toponymie est fortement représentée dans la littérature onomastique à portée politique ${ }^{2}$, qu'il s'agisse d'ouvrages ou de dossiers à visée générale (Boyer, Paveau, 2008; Guillorel, 2008) ou de travaux plus spécifiques portant sur les noms de continents ou d'ensembles géopolitiques3, de pays 4 , de régions ${ }^{5}$, de villes ou de villages ${ }^{6}$, de quartiers 7 , de rues ${ }^{8}$, ou plus largement sur la toponymie urbaine (Bouvier, Guillon, 2001), mais aussi sur les noms d'espaces naturels (Pelletier, 2007).

Parfois traitée concurremment à la toponymie9, l'anthroponymie et l'ethnonymie sont également très présentes, notamment dans une perspective diachronique ${ }^{10}$, qu'elle porte sur les dénominations individuelles ${ }^{11}$ ou collectives ${ }^{12}$, sur les dénominations officielles, officieuses ou polémiques (Boidin, 2007), et donc finalement sur les concurrences et les jeux de dénominations.

Les autres catégories de noms propres - leur classement n'allant d'ailleurs pas de soi - n'ont fait l'objet que de travaux plus rares, et souvent plus récents. Ainsi en va-t-il des noms de périodes ou chrononymes (Bacot, Douzou, Honoré, 2008) ou encore des noms d'actions: noms de guerres (Perraki, 1990 ; Veniard, dans Cassanas et al., 2004; Veniard dans Lecolle et al., 2009), de batailles (Paveau, dans Boyer, Paveau, 2008 et dans Lecolle et al., 2009), d'événements ${ }^{13}$, de négociations (Barbet, 2009).

La dimension politique de certains noms propres tient souvent au fait que la

2. Même si nous nous garderons bien dans ce texte de toute prétention à l'exhaustivité, nous avons choisi de fournir un assez grand nombre de références, et pour rester dans le format imparti, nous avons dû renoncer à indiquer les titres des articles de revues et contributions à ouvrages collectifs lorsque ces revues ou ouvrages sont référencés par ailleurs en tant que tels.

3. Tournier, dans Honoré, Paveau, Périès, 2000 ; Faure, dans Beillevaire, Honoré, 2001; Siblot, dans Bacot, Rémi-Giraud, 2007 ; Cortier, ibid.

4. Weber, dans Nora, 1986; Weis, 1993; Fiala, 1996; Bizet, 2003; Tissier, 2004 ; Pineira-Tresmontant, 2004 ; Cislaru, dans Bacot, Rémi-Giraud, 2007.

5. Gardy, 1987; Regault, 1996; Darot, 1997; Beillevaire, dans Beillevaire, Honoré, 2001.

6. Henry, 1985; Leimdorfer, 2001; 2005; Frizzi, 2005; Delattre, 2007 ; Yasri-Labrique, 2008.

7. Saunier, 1993; Bocquet, dans Bouvier, Guillon, 2001 ; Barberis, dans Bacot, Rémi-Giraud, 2007.

8. Milo, dans Nora, 1986; Saunier, 1990 ; Bacot, 1991; Bauer, 2001 ; Richard, dans Bouvier, Guillon, 2001 ; Gonac'h, 2007.

9. Cheriguen, 1994; Akin, 1999; Atoui, Benramdane, 2005; Cislaru, 2006; Basset, dans Bacot, Rémi-Giraud, 2007.

10. Bideau, Ducreux, Dupâquier, 1984; Cheriguen, 1987; 1998 ; Honoré, dans Honoré, Paveau, Périès, 2000 ; Durand, 2002.

11. Weitman, 1987; Le Bart, dans Honoré, Paveau, Périès, 2000 ; Guilhaumou, 2000.

12. Périès, 1992 ; Pauleau, 1997; Tournier, 2000.

13. Amalvi, dans Nora, 1984; Fragnon, 2007 ; Lecolle, dans Lecolle et al., 2009; Krieg-Planque, ibid. 
réalité qu'ils désignent appartient socialement au domaine de l'activité politicienne, dans sa double dimension de gestion de la chose publique et de compétition pour les postes de pouvoir. C'est notamment le cas des noms d'organisations et d'institutions. Michel Offerlé a proposé un cadre d'analyse riche en perspectives pour l'étude des noms de partis politiques (Offerlé, 1987), après qu'avait été interrogée pour la première fois leur morphologie (Tournier, 1981). Depuis lors, si l'interrogation a été souvent reprise, rares sont les travaux consacrés à l'onomastique partisane (Leroux, 2001; Fichard, dans Dufour et al., 2005; Tournier, 2010), ou encore aux noms propres des organisations syndicales (Pernot, dans Bacot, Rémi-Giraud, 2007), des alliances politiques (Pérus, 1987; Fiala, Rennes, 2002), des listes de candidats (Fiala, 2002; Vidal, 2004).

L'étude des dénominations d'institutions porte notamment quant à elle sur les noms d'assemblées ou agoronymes (Bacot, 2009) et sur les noms de ministères (Muller, 1999). On a aussi commencé à s'intéresser aux dénominations des textes juridiques, comme par exemple celles des constitutions (Bellescize, 1993), mais aussi des régimes politiques (Corcuff, 2005), des mythes politiques (Tournier, 1989) ou encore des politiques publiques (Pires, 2007).

\section{Les nouvelles problématiques de l'onomastique}

Les développements de l'onomastique générale durant les récentes décennies (Honoré, Paveau, Périès, 2000 ; Leroy, 2004; Vaxelaire, 2009) s'avèrent tout particulièrement intéressants pour les noms propres en politique. D'abord parce que le renouveau d'intérêt porté à l'objet et les nouvelles approches mobilisées se proposent de saisir le nom propre en discours - c'est-à-dire dans la «mise en œuvre de la langue par un sujet parlant», au sein d'une phrase ou d'un ensemble d'énoncés plus large (Gary-Prieur, dans Lecolle et al., 2009, p.154) - et non plus seulement en langue (Dufour, Dutilleul-Guerroudj, Laurent, 2005 ; Lecolle et al., 2009). Or, c'est bien toujours en discours que le nom propre se manifeste comme politique - et, d'une certaine façon, comme nom propre.

Ensuite, parce que ces nouvelles approches replacent volontiers la dénomination dans une perspective dialogique (Cassanas et al., 2004). Or, la dimension nécessairement conflictuelle du politique est inséparable d'une réalisation dans et par le dialogue, notamment mais pas seulement lors des débats institués comme tels.

Enfin, la remise en cause de l'asémantisme du nom propre au profit d'une recherche de son sens (Kleiber, 1981) ou de sa "signifiance» (Siblot, 1987) se révèle particulièrement porteuse s'agissant par exemple des noms de personnes ou de lieux (Lecolle, 2006), et surtout d'institutions ou d'organisations, mais aussi de périodes ou d'événements, surtout lorsque le processus de dénomination entre clairement dans des stratégies plus ou moins explicites: 
les baptêmes en politique sont le plus souvent datables et localisables, on peut rechercher qui en ont été les principaux acteurs - décideurs, politiciens, journalistes, mais aussi savants (Christin, dans Bacot et al., 2008) - et l'on peut donc voir dans le choix d'une dénomination propriale des intentions de légitimation, d'argumentation, de mobilisation (Bacot et al., 2008; Sommerer, ibid. ; Bacot, 2009).

Ces trois dimensions (discursive, dialogique, sémantique) n'en font en réalité qu'une seule : ces études portent bien sur les usages multiples des noms propres, et s'avèrent particulièrement utiles s'agissant de ces noms propres polylexicaux appelés « descriptifs » et «mixtes » (Jonasson, 1994) ou « dénominations propres» (Bosredon, Tamba, 1995; Veniard, dans Lecolle et al., 2009), comme par exemple les noms de partis ou d'assemblées. On est donc loin de la seule volonté d'identifier et de distinguer par le moyen d'une étiquette vide de sens - encore que la seule existence d'un nom quel qu'il soit vaut déjà reconnaissance de la réalité dénommée (Cheriguen, 1998). Avant même d'être pris dans le discours, le nom propre à caractère politique - ou utilisé politiquement - est en soi un discours.

Pour autant, une fois le nom choisi et attribué, de multiples locuteurs vont s'en saisir comme de tout autre nom propre. Les travaux les plus récents s'intéressent donc non seulement aux conditions sociales (Lavelle, dans Bacot et al., 2008) et aux modalités linguistiques de sa constitution - néologie, composition, emprunt - mais aussi à ses modifications morphologiques - troncation, siglaison, dérivation, traduction (ou calque) (Pérus, 1987) - ou syntaxiques - modification - et à ses manipulations sémantiques - polysémie ou polyréférentialité ${ }^{14}$, détournement (Pires, 2007), métonymie (Lecolle, 2001; 2004; Pochard, dans Bacot, Rémi-Giraud, 2007), métaphore (Bacot, Rémi-Giraud, 2007), antonomase ${ }^{15}$. À ces considérations principalement linguistiques, s'en ajoutent d'autres liées à l'évolution de nos sociétés - et notamment à celle de leur sphère politique - vers un marketing envahissant et une juridicisation croissante : le nom propre est perçu comme une ressource appropriable qu'il convient de défendre, ou d'attaquer (Lefebvre-Teillard, dans Honoré, Paveau, 2000 ; Frizzi, 2005). Au croisement de ces diverses interrogations, se trouvent les études en matière de changements de dénominations - qu'il s'agisse de personnes, de collectivités, d'institutions ou d'organisations ${ }^{16}$.

Pour une part, dans la pratique discursive politique, il en va du nom propre comme du nom commun. Le même nom peut ne pas être entendu de la même façon par tout le monde ou dans tous les contextes : on jouera de la polysémie

14. Pernot, 2002 ; Lettieri, dans Bacot et al., 2008; Fragnon, Lamy, ibid.; Paveau, dans Boyer, Paveau, 2008.

15. Leroy, Siblot, dans Honoré et al., 2000 ; Decimo, Fiala, 2004 ; Fragnon, 2007; Barbet, 2009.

16. Weis, 1993; Wauthion, 1998; Guerrin, dans Akin, 1999; Honoré, 2000 ; Delattre, 2007 ; Gonac’h, 2007. 
pour rassembler le plus largement possible, au risque de la confusion. Deux noms différents peuvent avoir le même référent, mais on s'efforcera de leur attribuer des sens distincts ici et là : la synonymie ${ }^{17}$ produira alors de la distinction pour mobiliser son camp contre le camp adverse, au risque cette foisci de l'artificialité.

\section{Les usages politiques du nom propre}

Finalement, que fait-on quand on donne ou qu'on utilise, en politique ou de façon politique, un nom propre pour parler d'un objet, de quelque nature qu'il soit - personne, groupe, lieu, période, institution, organisation, événement?

D'abord, bien sûr, on pointe une portion de la réalité - qu'en quelque sorte on découpe - et on la présente comme spécifique : la dénomination propriale sert avant tout à procéder à cette sélection et à afficher cette singularité, et par là même s'oppose à l'usage du nom commun qui ne fait que classer une entité dans une catégorie ${ }^{18}$. L'usage du nom propre est inséparable d'une procédure de découpage et d'une stratégie de distinction. Mais paradoxalement, cette mise en exception ne trouve sa raison d'être politique qu'en cela qu'elle permet la revendication d'unité ou de représentativité pour construire un camp, pour donner à voir un clivage, pour unir et opposer. C'est parce que le «panache blanc » est unique qu'on peut s'y « rallier »...

S'il en est ainsi, c'est que le choix du nom ou le fait de l'utiliser contribue à légitimer ou à délégitimer, et donc à argumenter, à convaincre et à imposer (Bacot, 2010). Nous l'avons dit, le nom propre est en soi un discours, il est un récit qui nous parle de ce qu'il dénomme, évoquant un certain passé vu d'une certaine façon, voire un certain futur, dessinant un environnement, suggérant d'autres entités en relation avec celle qui est dénommée, esquissant sa description : le nom propre n'est politique que s'il n'est pas arbitraire, que s'il est porteur d'un message, que s'il ne se contente pas de désigner l'objet qu'il présente comme unique, mais dit quelque chose à son sujet - quelque chose qui lui fait jouer un rôle dans la conflictualité sociale. Tant il est vrai que « l'activité de nomination [...] sert moins à désigner des fragments de réalité qu'à les sémiotiser en fonction de l'expérience sociale des locuteurs " (Branca-Rosoff, 2007, p. 14).

Mais quelquefois, la dénomination propriale ne nous parle pas, ou pas seulement, du dénommé immédiat, mais d’un dénommé antérieur, déjà porteur du nom. Il en est ainsi quand on attribue à $X$ le nom de $Y$ - comme en patrotoponymie (Martel, dans Bouvier, Guillon, 2001), dans le cas de l'hommage à une personnalité dont on donne le nom à une rue. Le processus peut nous dire

17. Polysémie et synonymie doivent être pris ici dans un sens large.

18. Jean-Louis Vaxelaire souligne qu'un chevalier peut donner un nom à son épée, mais qu'il est plus rare que nous en donnions un à nos couteaux de cuisine (Vaxelaire, 2009, p. 113). 
l'importance que l'on accorde à cette voie, mais surtout, elle nous dit l'importance que l'on accorde à la personne honorée et sa caractéristique mise en avant à travers la modalité retenue de sa dénomination (Bacot, 1991; Bertrand, dans Bouvier, Guillon, 2001).

Pour autant, il ne faut jamais oublier qu'au-delà du récit dont les baptêmes chargent le nom choisi, il y a les récits qu'entendront de multiples autres acteurs quand ils le liront ou l'entendront, et les récits que ces acteurs voudront faire circuler quand ils l'utiliseront. Tant il est vrai que si le nom propre, en politique, nous parle de ce qu'il dénomme, il nous parle aussi de ceux qui l'ont adopté et de ceux qui en font usage. S'il ne classe pas ce qu'il dénomme, il classe par contre plus ou moins ceux qui l'emploient, qui refusent de l'employer ou qui le contestent, par exemple par quelque modalisation autonymique comme l'utilisation de guillemets. Ces comportements langagiers s'expliqueront par des considérations d'ordre cognitif et stratégique.

Ainsi, le nom propre en politique n'est-il jamais qu'une manière de dire la réalité d'une façon conflictuelle - et donc en même temps consensuelle, puisque tout clivage rassemble en même temps qu'il sépare.

\section{Références}

Agulhon M., 2007, Les mots de la République, Toulouse, Presses universitaires du Mirail. AKIN S. éd., 1999, Noms et re-noms. La dénomination des personnes, des langues et des territoires, Rouen, Publications de l'université de Rouen.

Atou B., Benramdane F. éd., 2005, Toponymie et anthroponymie de l'Algérie. Recueil bibliographique général, Oran, Crasc.

BACOT P., 1991, "L'Affaire Claude Bernard”. De quelques hommages publics à une illustration scientifique et de leur politisation », La nécessité de Claude Bernard, M. Jacques éd., Paris, Méridiens-Klincksieck, p.199-228.

- 2009, "Parlement, Région, Sécurité. Les noms des institutions parlementaires internationales régionales», Régionalisme et sécurité internationale, $\mathrm{H}$. HassanYari, A. Ousman éd., Bruxelles, Bruylant, p. 11-32.

- 2010, "A legitimação pela denominação. $O$ agorônimo como discurso argumentativo », Análises do discurso hoje, vol. III, I. L. Machado, R. de Mello éd. et trad., Rio de Janeiro, Editora Nova Fronteira, p. 11-35.

Bacot P., RÉmi-GIRAud S. éd., 2007, Mots de l'espace et conflictualité sociale, Paris, L'Harmattan.

Bacot P., Douzou L., Honoré J.-P. éd., 2008, Mots. Les langages du politique, nº 87, juin, Chrononymes. La politisation du temps.

Barbet D., 2009, Grenelle. Histoire politique d'un mot, Rennes, Presses universitaires de Rennes.

BAUER L., 2001, «De la diachronie à la synchronie. Étude de la dénomination des voies à Cergy-Pontoise », Langage et société, nº 96, juin, p. 9-27. 
Bellescize D., 1993, «De l'Acte additionnel aux Constitutions de l'Empire (22 avril 1815). Une Constitution mal nommée pour un régime sans nom», Revue de droit public et de science politique, $\mathrm{n}^{\circ}$ 4, août, p. 1043-1077.

Beillevaire P., Honoré J.-P. éd., 2001, Mots. Les langages du politique, nº66, juillet, Discours d'Asie. Identités et ruptures.

Bideau A., Ducreux M.-E., DupÂQUIER J. éd., 1984, Le prénom. Mode et histoire, Entretiens de Mahler (1980), Paris, EHESS.

Bizet A., 2003, "Timor, l'orientale», Mots. Les langages du politique, n 71 , mars, p. $157-166$.

BoIDIN C., 2007, « Du Gran Líder Stoessner (1954-1989) au Karai Tendota Nicanor Duarte (2003-2006)», Mots. Les langages du politique, n०85, novembre, p.11-22.

Bosredon B., TAMBA I., 1995, "Titres de tableaux et noms propres», Nom propre et nomination, M. Noailly éd., Paris, Klincksieck, p.124-135.

Bouvier J.-C., GUILLon J.-M., 2001, La toponymie urbaine. Significations et enjeux, Paris, L'Harmattan.

Boyer H., Paveau M.-A. éd., 2008, Mots. Les langages du politique, n86, février, Toponymes. Instruments et enjeux.

BRANCA-RosofF S., 2007, "Approche discursive de la nomination/dénomination ", L'acte de nommer. Une dynamique entre langue et discours, G. Cislaru et al., Paris, Presses de la Sorbonne nouvelle, p.13-22.

Cassanas A., Demange A., Laurent B., LeCler A. éd., 2004, Dialogisme et nomination, Montpellier, Presses universitaires de Montpellier 3.

Cheriguen F., 1987, "Barbaros ou Amazigh. Ethnonymes et histoire politique en Afrique du Nord », Mots. Les langages du politique, $\mathrm{n}^{\circ}$ 15, octobre, p.7-22.

- 1994, "Anthropo-toponymie et désignation de l'“environnement politique" ", Mots. Les langages du politique, $\mathrm{n}^{\circ} 39$, juin, p. 93-98.

- 1998, «Nommer pour exister. De l'ethnonyme comme enjeu politique», Mots. Les langages du politique, $\mathrm{n}^{\circ}$ 57, décembre, p. 29-37.

CISLARU G., 2006, «Nom de pays, nom de peuple. Quels usages, quelles identités?», Cahiers de sociolinguistique, $\mathrm{n}^{\circ} 11, \mathrm{p} .131-144$.

Corcuff S., 2005, «Taïwan existe-t-elle? III. Du débat constitutionnel. Nom de régime et territoire national », Monde chinois, été-automne, $\mathrm{n}^{\circ}$ 5, p. 7-17.

DAROT M., 1997, «Calédonie, Kanaky ou Caillou? Implicites identitaires dans la désignation de la Nouvelle-Calédonie», Mots. Les langages du politique, nº 53, décembre, p. 8-25.

DÉCIMO M., FialA P., 2004, «Michel Bréal, le marathon, l'olympisme et la paix», Mots. Les langages du politique, nº 76, novembre, p. 127-135.

DelatTRE E., 2007, «Le changement de nom des communes françaises. Aspects économiques, marketing et stratégiques », Revue d'économie régionale et urbaine, $\mathrm{n}^{0} 2$, p. 269-291.

Dufour F., Dutilleul-Guerroud E., LAurent B., 2005, La nomination. Quelles problématiques, quelles orientations, quelles applications?, Montpellier, Presses universitaires de la Méditerranée.

DulucQ S., Klein J.-F., Stora B., 2008, Les mots de la colonisation, Toulouse, Presses universitaires du Mirail. 
DURAND G., 2002, «Survivance des patronymes d'origine africaine à la Martinique chez les esclaves et les affranchis avant et après l'abolition de 1848 ", Nouvelle revue d'onomastique, no39-40, p. 247-305.

FialA P., 1996, «Ex-Yougoslavie, les noms aussi sont morcelés», Mots. Les langages du politique, $n^{\circ} 47$, juin, p. 144-147.

- 2002, «Motivés, motivé», Mots. Les langages du politique, nº 70, novembre, p. 133-137.

Fiala P., RenNes J., 2002, « Majorité plurielle. Trajectoire d'une formule», Mots. Les langages du politique, n68, mars, p. 213-130.

FRAGNON J., 2007, «Quand le 11-Septembre s'approprie le onze septembre. Entre dérive métonymique et antonomase», Mots. Les langages du politique, n 85 , novembre, p. 83-95.

FRIZzI G., 2005, «La défense du nom des collectivités territoriales et le droit à la propriété intellectuelle», Revue générale des collectivités territoriales, nº 32 (1-2), p. 3-27.

GARDY P., 1987, «Réflexions à propos d'un toponyme : Larzac. Le nom impropre?», Cahiers de praxématique, n 8, p. 67-76.

GonAC'H J., 2007, «Pratiques de redénomination des rues à Vitrolles», L'acte de nommer. Une dynamique entre langues et discours, G. Cislaru et al., Paris, Presses de la Sorbonne nouvelle, p. 87-100.

Guilhaumou J., 2000, "Un nom propre en politique : Sieyès », Mots. Les langages du politique, $\mathrm{n}^{\circ} 63$, juillet, p. 74-86.

GUILLOREL H. éd., 2008, Toponymie et politique. Les marqueurs linguistiques du territoire, Bruxelles, Bruylant.

HenRY M., 1985, "La production praxémique d'un toponyme : Ladrecht», Cahiers de praxématique, $\mathrm{n}^{\circ} 4$, p. 41-64.

Honoré J.-P., PAVEAu M.-A., PÉrIÈs G. éd., 2000, Mots. Les langages du politique, no63, juillet, Noms propres.

JONASSON K., 1994, Le nom propre. Constructions et interprétations, Louvain-la-Neuve, Duculot.

KLeIB ER G., 1981, Problèmes de référence. Descriptions définies et noms propres, Paris, Klincksieck.

LeCOLLE M., 2001, «Personnification et métonymie dans la presse écrite. Comment les différencier?», Semen, nº 15 (2), p.97-112.

- 2004, «Toponymes en jeu. Diversité et mixage des emplois métonymiques de toponymes », Studii si cercercerati filologica, nº 3, p. 5-13.

- 2006, "Polyvalence des toponymes et interprétation en contexte», Pratiques, no 129-130, p.107-122.

Lecolle M., Paveau M.-A., Reboul-Touré S. éd., 2009, Carnets du Cediscor, nº11, Le nom propre en discours, Paris, Presses de la Sorbonne nouvelle.

LEIMdORFER F., 2005, «Des villes, des mots, des discours », Langage et société, nº114, p.129-146.

- 2001, "Saint-Quentin en Yvelines" ou "Ville nouvelle" ? Le choix de nom comme choix de ville», Langage et société, nº 96, p. 71-103.

LEROUX F., 2001, «Pour une onomastique des partis politiques. Éléments d'une étude des dénominations partisanes », Mémoire de l’IEP de Paris. 
LEROY S., 2004, Le nom propre en français, Paris, Gap, Ophrys.

MULLer P., 1999, «D’Instruction publique à Éducation nationale», Mots. Les langages du politique, n61, décembre, p.149-156.

NorA P. éd., 1984, 1986, 1992, Les lieux de mémoire, Paris, Gallimard (trois volumes).

OFFERLÉ M., 1987, Les partis politiques, Paris, PUF (Que sais-je ?).

Pauleau C., 1997, "Calédonien et Caldoche», Mots. Les langages du politique, nº53, décembre, p. 48-65.

Pelletier Ph., 2007, Le Japon. Géographie, géopolitique et géohistoire, Paris, SEDES.

PÉrIÈs G., 1992, "L'Arabe, le Musulman, l'Ennemi dans le discours militaire de la “guerre révolutionnaire" pendant la guerre d’Algérie », Mots. Les langages du politique, $\mathrm{n}^{\circ} 30$, mars, p. 53-70.

Pernot H., 2002, "L'appropriation du mot sud par les militants Sud-PTT», Mots. Les langages du politique, nº68, mars, p. 109-121.

PerRAKI V., 1990, "Du non-dit au cliché. Les avatars de Grande guerre et de Guerre mondiale», Mots. Les langages du politique, $\mathrm{n}^{0} 24$, septembre, p. 5-19.

PÉRUS J., 1987, «Un emprunt au russe dans la terminologie politique des années trente: front populaire», Mots. Les langages du politique, n015, octobre, p. 189-192.

Pineira-Tresmontant C., 2004, "Sémantique et histoire. España et Españas dans le discours de Juan Carlos », Mots. Les langages du politique, nº 75, juillet, p. 61-73.

PIRES M., 2007, «Le détournement de sigle. Le cas de CPE», Langage et société, n ${ }^{121-}$ 122, septembre-décembre, p. 289-303.

Regault J.-M., 1996, «Tahiti, EFO, Polynésie française, Ao Maohi... Quel nom pour ce territoire d'Outre-Mer? Un essai d'onomastique politique », Revue juridique et politique. Indépendance et coopération, n³ 3, p. 296-309.

SAUNIER P.-Y., 1990, «Tempêtes dans une petite plaque d'émail bleu. Les noms de rue à Lyon entre 1814 et 1914 ", Cahiers d'histoire, nº 4, p. 133-154.

- 1993, "La ville et ses découpages », Mélanges de l'École française de Rome. Italie et Méditerranée, no 105 (2), p. 375-403.

Siвlot P., 1987, «De la signifiance du nom propre», Cahiers de praxématique, n8, p. 197-114.

- 1989, "Noms propres et mains sales. De l'inscription des luttes sociales dans les praxèmes en nomination individuelle », Langages, n² 24-93, p. 64-83.

TISSIER Y., 2004 [2 édition], Dictionnaire de l'Europe. États d'hier et d'aujourd'hui de 1789 à nos jours, Paris, Vuibert.

TOURNIER M., 1981, «Vers une grammaire des dénominations socio-politiques au début de la Troisième République (1879-1905)», Mots. Les langages du politique, no 2, mars, p. 51-72.

- 1989, "Le Grand Soir, un mythe de fin de siècle », Mots. Les langages du politique, $\mathrm{n}^{\circ}$ 19, juin, p. 79-94.

- 2000, "Franc, stéréotype du Français», Mots. Les langages du politique, n64, décembre, p.136-141.

- 2007, Les mots de Mai 68, Toulouse, Presses universitaires du Mirail.

- 2010, Des noms et des gens en République (1879-1914), Paris, L'Harmattan.

VAN DE VELDE D., 2000, «Existe-t-il des noms propres de temps? », Lexique, nº 15, p. 35-45. 
VAXELAIRE J.-L., 2009, "Lexicologie du nom propre et onomastique», Nouvelle revue d'onomastique, $\mathrm{n}^{\circ}$ 51, p. 301-315.

VIDAL F., 2004, "Construction des identités et légitimation à travers les titres des listes candidates aux élections municipales à Lyon sous la Ve République», Mémoire de DEA de science politique, Institut d'études politiques de Lyon.

WAUthION M., 1998, "Noms de pays. Le nom changeant de ses institutions », Revue nouvelle, $\mathrm{n}^{0} 107$ (2), p. 49-65.

WEIS B., 1993, «Essai sur la francisation des toponymes en Alsace depuis la royauté jusqu'à nos jours », Nouvelle revue d'onomastique, n² 21-22, p. 109-112.

Weitman S., 1987, «Prénoms et orientations nationales en Israël, 1882-1980 », Annales. Histoire, Sciences sociales, no 42 (4), p. 879-900.

YASRI-LABRIQUE E., 2008, "La Turquie, terre eurasiatique et république bicéphale. Ankara et Istanbul à travers la presse », Mots. Les langages du politique, no 86 , février, p. 37-52. 